\title{
Topology of Lattice Gauge Fields *
}

\author{
M. Lüscher \\ Institut für Theoretische Physik, Universität Bern, CH-3012 Bern, Switzerland
}

\begin{abstract}
Non-Abelian gauge fields on a four-dimensional hypercubic lattice with small action density $[\operatorname{Tr}\{1-U(\dot{p})\}<0.03$ for $\mathrm{SU}(2)$ gauge fields] are shown to carry an integer topological charge $Q$, which is invariant under continuous deformations of the field. A concrete expression for $Q$ is given and it is verified that $Q$ reduces to the familiar Chern number in the classical continuum limit.
\end{abstract}

\section{Introduction}

Differentiable SU(2) ${ }^{1}$ gauge fields $A_{\mu}=-A_{\mu}^{+}$on a four-dimensional torus $T^{4}$ ( = finite volume with periodic boundary conditions) carry a topological number [1]

$$
\begin{gathered}
Q=-\frac{1}{16 \pi^{2}} \int_{T^{4}} d^{4} x \operatorname{Tr}\left\{F_{\mu \nu}{ }^{*} F_{\mu \nu}\right\}, \\
F_{\mu \nu}=\partial_{\mu} A_{\nu}-\partial_{\nu} A_{\mu}+\left[A_{\mu}, A_{\nu}\right] ; \quad{ }^{*} F_{\mu \nu}=\frac{1}{2} \varepsilon_{\mu \nu \varrho \sigma} F_{\varrho \sigma},
\end{gathered}
$$

which assumes integer values only. One expects that this structure gives rise to interesting effects at the quantum level, in particular, the topological susceptibility

$$
\chi_{t}=\left\langle Q^{2}\right\rangle / \text { Volume }
$$

enters the chiral Ward identities and may be responsible for the large mass of the $\eta^{\prime}$ meson (see [2] for a recent discussion).

On a lattice, continuity (in space) is lost and any lattice gauge field $U(n, \mu)[3]$ can be continuously deformed to the trivial field $U(n, \mu)=1$ : there is apparently no topological structure. However, this argumentation overlooks the fact that ultimately one is only interested in lattice gauge theories with small values of the bare coupling constant $g$, i.e. in the region near the (quantum) continuum limit. In

* Work supported in part by Schweizerischer Nationalfonds

1 For clarity, the gauge group is taken to be $\mathrm{SU}(2)$ throughout the paper. The results can easily be generalized to any compact gauge group 
this regime most of the fields contributing to the functional integral have a small lattice action density, because

$$
\langle\operatorname{Tr}\{1-U(\dot{p})\}\rangle=\frac{3}{8} g^{2}+O\left(g^{4}\right),
$$

$U(\dot{p})$ : parallel transporter around the plaquette $p$.

Without affecting the continuum limit one could even restrict the domain of integration in the functional integral to fields with

$$
\operatorname{Tr}\{1-U(\dot{p})\}<\varepsilon,
$$

where $\varepsilon$ is any small positive number independent of $g$ and the size of the lattice. This condition imposes some continuity (in space) on the fields in the sense that parallel transporters $U(\mathscr{C})$ around closed loops $\mathscr{C}$ on the lattice are near 1 for small loops. It is therefore not surprising (and will be proved in this article) that such fields again fall into disconnected topological classes, provided $\varepsilon$ is small enough, i.e. $\varepsilon \leqq 0.03^{2}$. In this way, topology is recovered from lattice gauge theories in both the classical and the quantum continuum limit.

In order to make topological quantities like $\chi_{t}$ calculable in lattice gauge theories, a definition of a topological charge $Q$ for lattice gauge fields will be proposed (Sect. 3). $Q$ has the following properties:

(i) $Q$ is defined for all fields on a hypercubic lattice with periodic boundary conditions except for a singular set of fields, which has zero measure in the functional integral. Fields for which $Q$ is not defined, are called exceptional.

(ii) Fields with $\operatorname{Tr}\{1-U(\dot{p})\}<0.03$ for all plaquettes $p$ are not exceptional.

(iii) $Q$ assumes integer values only and does not change, when the field is continuously deformed keeping away from the exceptional configurations.

(iv) $Q$ has the form

$$
Q=\sum_{n} q(n),
$$

where $n$ runs over the vertices of the lattice and the charge density $q(n)$ is a local function of the lattice gauge field.

(v) In the classical continuum limit, the charge density $q(n)$ approaches the familiar form

$$
q(n)=-\frac{a^{4}}{16 \pi^{2}} \operatorname{Tr}\left\{F_{\mu \nu}(a n) * F_{\mu \nu}(a n)\right\}+O\left(a^{5}\right),
$$

where $a$ is the lattice spacing [cf. Eq. (1)].

The existence of the charge $Q$ immediately implies that, as asserted above, fields with $\operatorname{Tr}\{1-U(\dot{p})\}<0.03$ cannot always be deformed into the "vacuum" configuration $U(n, \mu)=1$ without passing through configurations with $\operatorname{Tr}\{1-U(\dot{p})\}>0.03$ for some $p$.

A lattice topological number with properties as listed above has already been found for two-dimensional spin systems [4]. The construction given here is similar, but rather less obvious geometrically. The reason is that the geometrical object, which carries the topological charge in the gauge field case, really is the 
principal bundle on which $A_{\mu}$ lives, and this is not easily visualized. In Sect. 2 the notion and topology of a principal bundle on a torus is therefore first discussed. Then, associating a principal bundle to any lattice gauge field, one obtains a natural definition of $Q$ by identifying it with the topological charge of the bundle (Sect. 3). The stated properties of $Q$ are verified in Sect. 4 and some concluding remarks are made in the final Sect. 5.

\section{Topology of SU(2) Principal Bundles on a Torus $T^{4}$}

It is convenient to think of a four-dimensional torus $T^{4}$ as being $\mathbb{R}^{4}$, where any two points $x$ and $y$ are identified, if

$$
\frac{1}{L}\left(x_{\mu}-y_{\mu}\right) \in \mathbb{Z} \text { for all } \mu=1,2,3,4 .
$$

The value of the period $L$ is irrelevant for topological considerations. It is here taken to be a natural number so that we can divide $T^{4}$ into unit cells $c(n)\left(n \in \mathbb{Z}^{4}\right)$ :

$$
c(n)=\left\{x \in \mathbb{R}^{4} \mid 0 \leqq\left(x_{\mu}-n_{\mu}\right) \leqq 1 \text { for all } \mu\right\} .
$$

If $L \geqq 2$, these cells are contractible pieces of $T^{4}$ so that any bundle over $T^{4}$ is trivial, when restricted to $c(n)$ (for the definition of a principal bundle, see e.g. [5, p. 50ff.]). The bundle is then characterized by the transition matrices $v_{n, \mu}$, which tell how to go from $c(n)$ to the neighboring cell $c(n-\hat{\mu})^{3}$. Thus, $v_{n, \mu}$ is a function defined on the face

$$
f(n, \mu)=\left\{x \in c(n) \mid x_{\mu}=n_{\mu}\right\}=c(n) \cap c(n-\hat{\mu})
$$

and taking values in SU(2). Since the bundle is defined over $T^{4}$, the transition matrices must respect periodicity, i.e. for any $m \in \mathbb{Z}^{4}$

$$
v_{n+m L, \mu}(x+m L)=v_{n, \mu}(x) \text {. }
$$

Furthermore, since the four cells $c(n), c(n-\hat{\mu}), c(n-\hat{v})$ and $c(n-\hat{\mu}-\hat{v})$ overlap along the plaquette

$$
p(n, \mu, v)=\left\{x \in c(n) \mid x_{\mu}=n_{\mu}, x_{v}=n_{v}\right\} \quad(\mu \neq v),
$$

the transition matrices must satisfy the cocycle condition

$$
v_{n-\hat{\mu}, v}(x) \cdot v_{n, \mu}(x)=v_{n-\hat{v}, \mu}(x) \cdot v_{n, v}(x)
$$

for all $x \in p(n, \mu, v)$. To sum up, an $\mathrm{SU}(2)$ principal bundle over $T^{4}$ is completely characterized by a set of functions $v_{n, \mu}(x), x \in f(n, \mu)$, with values in $\mathrm{SU}(2)$, such that Eqs. (9) and (11) hold.

SU(2) principal bundles over $T^{4}$ fall into topological classes labelled by an integer topological charge $Q$. An explicit expression for $Q$ in terms of the transition

$3 \hat{\mu}$ denotes the unit vector in the positive $\mu$-direction 
matrices $v_{n, \mu}$ is

$$
\begin{aligned}
Q= & -\frac{1}{24 \pi^{2}} \sum_{n \in \Lambda} \sum_{\mu, v, \varrho, \sigma} \varepsilon_{\mu v \varrho \sigma}\left\{3 \int_{p(n, \mu, v)} d^{2} x \operatorname{Tr}\left[\left(v_{n, \mu} \partial_{\varrho} v_{n, \mu}^{-1}\right)\left(v_{n-\hat{\mu}, \nu}^{-1} \partial_{\sigma} v_{n-\hat{\mu}, v}\right)\right]\right. \\
& \left.+\int_{f(n, \mu)} d^{3} x \operatorname{Tr}\left[\left(v_{n, \mu}^{-1} \partial_{v} v_{n, \mu}\right)\left(v_{n, \mu}^{-1} \partial_{\varrho} v_{n, \mu}\right)\left(v_{n, \mu}^{-1} \partial_{\sigma} v_{n, \mu}\right)\right]\right\},
\end{aligned}
$$

where $\Lambda$ denotes the lattice

$$
\Lambda=\left\{n \in \mathbb{Z}^{4} \mid 1 \leqq n_{\mu} \leqq L \text { for all } \mu\right\} .
$$

It is not difficult to prove that the above $Q$ is an integer, which assumes different values for topologically inequivalent bundles. Namely, using the identity ${ }^{4}$

$$
\begin{aligned}
\varepsilon_{\mu \nu \varrho \sigma} & \operatorname{Tr}\left\{\left(w^{-1} v\right) \partial_{v}\left(v^{-1} w\right)\left(w^{-1} v\right) \partial_{\varrho}\left(v^{-1} w\right)\left(w^{-1} v\right) \partial_{\sigma}\left(v^{-1} w\right)\right\} \\
= & \varepsilon_{\mu \nu \varrho \sigma} \operatorname{Tr}\left\{\left(w^{-1} \partial_{v} w\right)\left(w^{-1} \partial_{\varrho} w\right)\left(w^{-1} \partial_{\sigma} w\right)\right. \\
& \left.-\left(v^{-1} \partial_{v} v\right)\left(v^{-1} \partial_{\varrho} v\right)\left(v^{-1} \partial_{\sigma} v\right)+3 \partial_{v}\left[\left(v \partial_{\varrho} v^{-1}\right)\left(w \partial_{\sigma} w^{-1}\right)\right]\right\},
\end{aligned}
$$

one first verifies that $Q$ is invariant under the gauge transformation

$$
v_{n, \mu}^{\prime}(x)=u_{n-\hat{\mu}}(x) v_{n, \mu}(x) u_{n}(x)^{-1}
$$

for any choice of differentiable $\mathrm{SU}(2)$-valued functions $u_{n}(x)(x \in c(n))$. One can then arrange the $u_{n}$ 's in such a way that all transition matrices $v_{n, \mu}^{\prime}$ are equal to one, except possibly those with $\mu=1$ and $n_{1}=0(\bmod L)$. Because of the cocycle condition (11), the remaining non-trivial transition matrices match along the plaquettes, thus making up a continuous, piecewise differentiable transition matrix $v(x)$ defined on the hyperplanes $x_{1}=m \cdot L(m \in \mathbb{Z})$. Due to periodicity, $\left.v(x)\right|_{x_{1}=L}$ really is a mapping from $S^{1} \times S^{1} \times S^{1}$ into $S U(2)$. Its winding number is characteristic for the topological class of the bundle and is in fact easily seen to be identical with $Q$.

\section{Definition of a Topological Charge $Q$ for Lattice Gauge Fields}

We here consider periodic SU(2) lattice gauge fields, i.e. for each bond with endpoints $n, n+\hat{\mu}$ on a hypercubic lattice $\mathbb{Z}^{4}$ we are given a parallel transporter $U(n, \mu) \in \mathrm{SU}(2)$ subject to

$$
U(n+m L, \mu)=U(n, \mu) \text { for all } m \in \mathbb{Z}^{4} .
$$

The lattice $\mathbb{Z}^{4}$ is thought to be imbedded in $\mathbb{R}^{4}$. Cells $c(n)$, faces $f(n, \mu)$ and plaquettes $p(n, \mu, v)$ are then defined as in Sect. 2. The topological charge $Q$ of the gauge field $U(n, \mu)$ will now be defined by first constructing a principal bundle (i.e. a set of transition matrices $v_{n, \mu}$ ) and then identifying $Q$ with the topological charge (12) of the bundle.

4 A sum over repeated indices is implied 


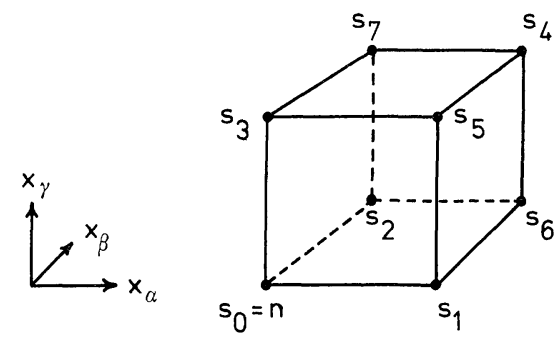

Fig. 1. Standard labelling of the corners of a face $f(n, \mu)$

The construction of the $v_{n, \mu}$ 's is not really difficult, but notationally voluminous. First, for each corner

$$
x=n+\sum_{\mu=1}^{4} z_{\mu} \hat{\mu}, \quad z_{\mu} \in\{0,1\},
$$

of the cell $c(n)$, one defines a standard parallel transporter from $x$ to $n^{5}$ :

$$
\begin{aligned}
w^{n}(x)= & U(n, \hat{1})^{z_{1}} U\left(n+z_{1} \hat{1}, \hat{2}\right)^{z_{2}} \\
& \cdot U\left(n+z_{1} \hat{1}+z_{2} \hat{2}, \hat{3}\right)^{z_{3}} U\left(n+z_{1} \hat{1}+z_{2} \hat{2}+z_{3} \hat{3}, \hat{4}\right)^{z_{4}} .
\end{aligned}
$$

For any two neighboring corners $x, y$ of $c(n)$ we furthermore set

$$
\begin{array}{ll}
u_{x y}^{n}=w^{n}(x) U(x, \mu) w^{n}(y)^{-1}, & \text { if } y=x+\hat{\mu}, \\
u_{x y}^{n}=\left(u_{y x}^{n}\right)^{-1}, & \text { if } y=x-\hat{\mu} .
\end{array}
$$

These matrices are parallel transporters along closed loops in $c(n)$ starting and ending at $n$. In particular, the trace of any product of such matrices is gauge invariant. One may interpret the $u_{x y}^{n}$ 's as the field variables in $c(n)$ in a complete axial gauge. Along the face $f(n, \mu)$ the gauges in $c(n)$ and $c(n-\hat{\mu})$ are related by transition matrices $v_{n, \mu}(x)$ :

$$
\begin{gathered}
u_{x y}^{n-\hat{\mu}}=v_{n, \mu}(x) u_{x y}^{n} v_{n, \mu}(y)^{-1}, \\
v_{n, \mu}(x) \stackrel{\text { def }}{=} w^{n-\hat{\mu}}(x) w^{n}(x)^{-1} .
\end{gathered}
$$

The transition matrices are periodic [Eq. (9)] and satisfy the cocycle relation (11), but so far they are only defined at the corners of the faces $f(n, \mu)$.

We now look for a smooth interpolation of $v_{n, \mu}(x)$ to all $x \in f(n, \mu)$. To this end, we first label the corners of $f(n, \mu)$ as follows (cf. Fig. 1). Let $\alpha, \beta, \gamma \in\{1,2,3,4\} \backslash\{\mu\}$ be the three indices complementary to $\mu$ such that $\alpha<\beta<\gamma$. Define $s_{0}, \ldots, s_{7}$ by

$$
\begin{array}{ll}
s_{0}=n ; & s_{4}=n+\hat{\alpha}+\hat{\beta}+\hat{\gamma} \\
s_{1}=n+\hat{\alpha} ; & s_{5}=n+\hat{\alpha}+\hat{\gamma} \\
s_{2}=n+\hat{\beta} ; & s_{6}=n+\hat{\alpha}+\hat{\beta} \\
s_{3}=n+\hat{\gamma} ; & s_{7}=n+\hat{\beta}+\hat{\gamma} .
\end{array}
$$

$5 \quad U(m, \mu)^{0}=1 ; U(m, \mu)^{1}=U(m, \mu)$ 
For $m=n$ or $m=n-\hat{\mu}$ and $x \in f(n, \mu)$, we furthermore define the following set of functions :

$$
\begin{aligned}
& f_{n, \mu}^{m}\left(x_{\gamma}\right)=\left(u_{s_{3} s_{0}}^{m}\right)^{y_{\gamma}}\left(u_{s_{0} s_{3}}^{m} u_{s_{3} s_{7}}^{m} u_{s_{7} s_{2}}^{m} u_{s_{2} s_{0}}^{m}\right)^{y_{\gamma}} \\
& \cdot u_{s_{0} S_{2}}^{m}\left(u_{s_{2} S_{7}}^{m}\right)^{y_{\gamma}} \\
& g_{n, \mu}^{m}\left(x_{\gamma}\right)=\left(u_{s_{5} s_{1}}^{m}\right)^{y_{\gamma}}\left(u_{s_{1} s_{5}}^{m} u_{s_{5} s_{4}}^{m} u_{s_{4} s_{6}}^{m} u_{s_{6} s_{1}}^{m}\right)^{y_{\gamma}} \\
& \cdot u_{s_{1} s_{6}}^{m}\left(u_{s_{6} s_{4}}^{m}\right)^{y_{\gamma}} \text {, } \\
& h_{n, \mu}^{m}\left(x_{\gamma}\right)=\left(u_{s_{3} s_{0}}^{m}\right)^{y_{\gamma}}\left(u_{s_{0} s_{3}}^{m} u_{s_{3} s_{5}}^{m} u_{s_{5} s_{1}}^{m} u_{s_{1} s_{0}}^{m}\right)^{y_{\gamma}} \\
& \cdot u_{s_{0} S_{1}}^{m}\left(u_{s_{1} s_{5}}^{m}\right)^{y_{\gamma}} \\
& k_{n, \mu}^{m}\left(x_{\gamma}\right)=\left(u_{s_{7} s_{2}}^{m}\right)^{y_{\gamma}}\left(u_{s_{2} s_{7}}^{m} u_{s_{7} s_{4}}^{m} u_{s_{4} s_{6}}^{m} u_{s_{6} s_{2}}^{m}\right)^{y_{\gamma}} \\
& \cdot u_{s_{2} s_{6}}^{m}\left(u_{s_{6} s_{4}}^{m}\right)^{y_{\gamma}} \\
& l_{n, \mu}^{m}\left(x_{\beta}, x_{\gamma}\right)=\left[f_{n, \mu}^{m}\left(x_{\gamma}\right)^{-1}\right]^{y_{\beta}}\left[f_{n, \mu}^{m}\left(x_{\gamma}\right) k_{n, \mu}^{m}\left(x_{\gamma}\right) g_{n, \mu}^{m}\left(x_{\gamma}\right)^{-1} h_{n, \mu}^{m}\left(x_{\gamma}\right)^{-1}\right]^{y_{\beta}} \\
& \cdot h_{n, \mu}^{m}\left(x_{\gamma}\right)\left[g_{n, \mu}^{m}\left(x_{\gamma}\right)\right]^{y_{\beta}}, \\
& S_{n, \mu}^{m}\left(x_{\alpha}, x_{\beta}, x_{\gamma}\right)=\left(u_{s_{0} S_{3}}^{m}\right)^{y_{\gamma}}\left[f_{n, \mu}^{m}\left(x_{\gamma}\right)\right]^{y_{\beta}}\left[l_{n, \mu}^{m}\left(x_{\beta}, x_{\gamma}\right)\right]^{y_{\alpha}} \text {. }
\end{aligned}
$$

Here, $y_{\lambda}=x_{\lambda}-n_{\lambda}(\lambda=\alpha, \beta, \gamma)$ varies between 0 and 1 and powers $u^{y}$ of matrices $u \in \mathrm{SU}(2)$ are defined as follows. If $u \neq-1$ there is a unique way to write

$$
u=\exp i \mathbf{t} \cdot \boldsymbol{\sigma} ; \boldsymbol{\sigma}: \text { Pauli-Matrices, }
$$

where $\mathbf{t}$ is real and $|\mathbf{t}|<\pi$. We then set $^{6}$

$$
u^{y}=\operatorname{expiyt} \cdot \boldsymbol{\sigma} .
$$

If $u=-1, u^{y}$ is not defined. The matrices (22) are therefore not defined for a set of exceptional lattice gauge fields $U(n, \mu)$. This is the origin of the exceptional configurations announced in the introduction. As can be seen from the above, they only make up a lower dimensional submanifold in the space of all lattice gauge fields.

We are now in a position to define a smooth interpolation of $v_{n, \mu}(x)$ to all $x \in f(n, \mu)$ :

$$
v_{n, \mu}(x) \stackrel{\text { def }}{=} S_{n, \mu}^{n-\hat{\mu}}(x)^{-1} v_{n, \mu}(\mathrm{n}) \mathrm{S}_{n, \mu}^{n}(x)
$$

For non-exceptional fields, $v_{n, \mu}(x)$ is a differentiable and periodic [Eq. (9)] function of $x \in f(n, \mu)$ with values in $\mathrm{SU}(2)$. It is also not difficult to verify that $v_{n, \mu}\left(s_{i}\right)$ $(i=0, \ldots, 7)$ as computed from Eq. (25) is equal to the corresponding transition matrix defined earlier [Eq. (20)]. The non-trivial feature of Eq. (25) is, however, that

Lemma. $v_{n, \mu}(x)$ is a cocycle, i.e. Eq. (11) holds.

6 In what follows, we only use that (i) $u^{0}=1, u^{1}=u$. (ii) $\left(u^{-1}\right)^{y}=\left(u^{y}\right)^{-1}$. (iii) $\left(v u v^{-1}\right)^{y}=v u^{y} v^{-1}$. Other definitions of $u^{y}$ would therefore be possible, e.g. $u^{y}=(1-y+y u) \operatorname{det}(1-y+y u)^{-1 / 2}$ 


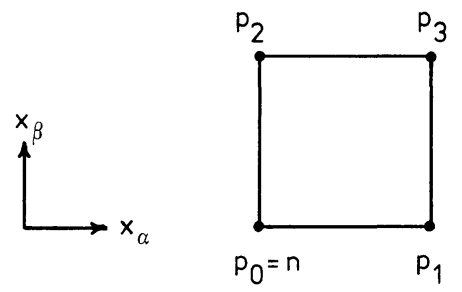

Fig. 2. Standard labelling of the corners of a plaquette $p(n, \mu, v)$

Proof. We have to evaluate $v_{n, \mu}$ along the plaquettes $p$ bounding $f(n, \mu)$. As for the faces, it is convenient to introduce a standard parametrization of the corners of the plaquettes $p(n, \mu, v)$ (see Fig. 2): choose $\alpha<\beta$ to be the indices complementary to $\mu$, $v$ and set

$$
p_{0}=n ; p_{1}=n+\hat{\alpha} ; p_{2}=n+\hat{\beta} ; p_{3}=n+\hat{\alpha}+\hat{\beta} .
$$

For $m=n, n-\hat{\mu}, n-\hat{v}$ or $n-\hat{\mu}-\hat{v}$ define furthermore

$$
\begin{aligned}
P_{n, \mu v}^{m}\left(x_{\alpha}, x_{\beta}\right)= & \left(u_{p_{0} p_{2}}^{m}\right)^{y_{\beta}} \\
& \cdot\left[\left(u_{p_{2} p_{0}}^{m}\right)^{y_{\beta}}\left(u_{p_{0} p_{2}}^{m} u_{p_{2} p_{3}}^{m} u_{p_{3} p_{1}}^{m} u_{p_{1} p_{0}}^{m}\right)^{y_{\beta}} u_{p_{0} p_{1}}^{m}\left(u_{p_{1} p_{3}}^{m}\right)^{y_{\beta}}\right]^{y_{\alpha}} .
\end{aligned}
$$

By explicit computation, starting from Eq. (22f), one then obtains $(\lambda=\alpha, \beta$ or $\gamma)$

$$
\begin{array}{ll}
S_{n, \mu}^{m}(x)=P_{n, \mu \lambda}^{m}(x) & \text { for } \quad x \in p(n, \mu, \lambda), \\
S_{n, \mu}^{m}(x)=R_{n, \mu ; \lambda}^{m}(x) P_{n+\hat{\lambda}, \mu \lambda}^{m}(x) & \text { for } \quad x \in p(n+\hat{\lambda}, \mu, \lambda),
\end{array}
$$

where

$$
\begin{aligned}
& R_{n, \mu ; \alpha}^{m}\left(x_{\beta}, x_{\gamma}\right)=\left[\left(u_{s_{0} s_{3}}^{m} u_{s_{3} S_{7}}^{m} u_{s_{7} s_{2}}^{m} u_{s_{2} s_{0}}^{m}\right)^{y_{\gamma}} u_{s_{0} s_{2}}^{m}\right. \\
& \cdot\left(u_{s_{2} s_{7}}^{m} u_{s_{7} s_{4}}^{m} u_{s_{4} S_{6}}^{m} u_{s_{6} S_{2}}^{m}\right)^{y_{\gamma}} u_{s_{2} s_{6}}^{m} u_{s_{6} s_{1}}^{m}\left(u_{s_{1} s_{6}}^{m} u_{s_{6} S_{4}}^{m} u_{s_{4} s_{5}}^{m} u_{s_{5} S_{1}}^{m}\right)^{y_{\gamma}} \\
& \left.\cdot u_{s_{1} s_{0}}^{m}\left(u_{s_{0} s_{1}}^{m} u_{s_{1} s_{5}}^{m} u_{s_{5} s_{3}}^{m} u_{s_{3} s_{0}}^{m}\right)^{y_{\nu}}\right]^{y_{\beta}}\left(u_{s_{0} s_{3}}^{m} u_{s_{3} s_{5}}^{m} u_{s_{5} s_{1}}^{m} u_{s_{1} s_{0}}^{m}\right)^{y_{\nu}} u_{s_{0} s_{1}}^{m} \text {, } \\
& R_{n, \mu ; \beta}^{m}\left(x_{\alpha}, x_{\gamma}\right)=\left(u_{s_{0} s_{3}}^{m} u_{s_{3} S_{7}}^{m} u_{s_{7} s_{2}}^{m} u_{s_{2} s_{0}}^{m}\right)^{y_{\gamma}} u_{s_{0} s_{2}}^{m}, \\
& R_{n, \mu ; \gamma}^{m}\left(x_{\alpha}, x_{\beta}\right)=u_{s_{0} s_{3}}^{m} .
\end{aligned}
$$

Using this, Eq. (19) and

$$
\left(v u v^{-1}\right)^{y}=v u^{y} v^{-1}
$$

one verifies that

$$
R_{n, \mu ; \lambda}^{n-\hat{\mu}}=v_{n, \mu}(n) R_{n, \mu ; \lambda}^{n} v_{n, \mu}(n+\hat{\lambda})^{-1} .
$$

For $x \in p(n, \mu, v)$ we thus have

$$
v_{n, \mu}(x)=P_{n, \mu \lambda}^{n-\hat{\mu}}(x)^{-1} v_{n, \mu}(n) P_{n, \mu \lambda}^{n}(x),
$$

and for $x \in p(n+\hat{\lambda}, \mu, \lambda)$

$$
v_{n, \mu}(x)=P_{n+\hat{\hat{\lambda}}, \mu \lambda}^{n-\hat{\lambda}}(x)^{-1} v_{n, \mu}(n+\hat{\lambda}) P_{n+\hat{\lambda}, \mu \lambda}^{n}(x) .
$$


It follows that along $p(n, \mu, v)$

$$
\begin{aligned}
& v_{n-\hat{\mu}, v} v_{n, \mu}=\left(P_{n, \mu \nu}^{n-\hat{\mu}-\hat{v}}\right)^{-1} v_{n-\hat{\mu}, v}(n) v_{n, \mu}(n) P_{n, \mu \nu}^{n}, \\
& v_{n-\hat{v}, \mu} v_{n, v}=\left(P_{n, \mu \nu}^{n-\hat{\mu}-\hat{v}}\right)^{-1} v_{n-\hat{v}, \mu}(n) v_{n, v}(n) P_{n, \mu v}^{n} .
\end{aligned}
$$

By Eq. (20), the cocycle condition (11) holds for $x=n$ so that, by the above, it is satisfied for all $x \in p(n, \mu, v)$.

Summarizing, we have associated an SU(2) principal bundle to any nonexceptional lattice gauge field. The topological charge $Q$ of the field is defined to be equal to the charge (12) of the bundle. This is a rather complicated expression, but it is explicit enough to establish the properties (i)-(v) announced in Sect. 1.

\section{Properties of the Topological Charge $Q$}

Number (i) and (iii) of the list of properties of $Q$ discussed in Sect. 1 are obvious from the definition of $Q$. Number (ii) is due to the fact that if all $U(\dot{p})$ 's are near one, so are the matrices $u_{x y}^{m}$ and consequently all the products in Eqs. (22a)-(22f). The powers $u^{y}$ involved are then well-defined, i.e. the configuration is not exceptional. To verify the remaining properties (iv) and (v) we first derive an expression for the charge density $q(n)$. Namely, inserting Eq. (25) into Eq. (12) and using the identities (14), (28a) and (28b) gives

$$
Q=\sum_{n \in \Lambda} q(n)
$$

$$
\begin{aligned}
& q(n)=\frac{1}{24 \pi^{2}} \sum_{\mu, v, \varrho, \sigma} \varepsilon_{\mu \nu \varrho \sigma} \\
& \text {. }\left\{3 \int_{p(n+\hat{\mu}+\hat{v}, \mu, \nu)} d^{2} x \operatorname{Tr}\left[P_{n+\hat{\mu}+\hat{v}, \mu \nu}^{n} \partial_{\varrho}\left(P_{n+\hat{\mu}+\hat{v}, \mu \nu}^{n}\right)^{-1}\left(R_{n+\hat{\mu}, \mu ; \nu}^{n}\right)^{-1} \partial_{\sigma} R_{n+\mu, \hat{\mu} ; \nu}^{n}\right]\right. \\
& -3 \int_{p(n+\hat{v}, \mu, v)} d^{2} x \operatorname{Tr}\left[P_{n+\hat{v}, \mu \nu}^{n} \partial_{\varrho}\left(P_{n+\hat{v}, \mu \nu}^{n}\right)^{-1}\left(R_{n, \mu ; \nu}^{n}\right)^{-1} \partial_{\sigma} R_{n, \mu ; \nu}^{n}\right] \\
& -\int_{f(n+\hat{\mu}, \mu)} d^{3} x \operatorname{Tr}\left[S_{n+\hat{\mu}, \mu}^{n} \partial_{\nu}\left(S_{n+\hat{\mu}, \mu}^{n}\right)^{-1} S_{n+\hat{\mu}, \mu}^{n} \partial_{\varrho}\left(S_{n+\mu, \mu}^{n}\right)^{-1} S_{n+\hat{\mu}, \mu}^{n} \partial_{\sigma}\left(S_{n+\hat{\mu}, \mu}^{n}\right)^{-1}\right] \\
& \left.+\int_{f(n, \mu)} d^{3} x \operatorname{Tr}\left[S_{n, \mu}^{n} \partial_{v}\left(S_{n, \mu}^{n}\right)^{-1} S_{n, \mu}^{n} \partial_{\varrho}\left(S_{n, \mu}^{n}\right)^{-1} S_{n, \mu}^{n} \partial_{\sigma}\left(S_{n, \mu}^{n}\right)^{-1}\right]\right\} .
\end{aligned}
$$

$q(n)$ thus depends on the variables $u_{x y}^{n}$ alone, i.e. it is a gauge invariant combination of the parallel transporters $U(m, \mu)$ in the cell $c(n)$.

In the classical continuum limit

$$
U(n, \mu)=T \exp a \int_{0}^{1} d t A_{\mu}(a n+(1-t) a \hat{\mu}), \quad a \rightarrow 0,
$$

where $A_{\mu}(z)$ is a differentiable gauge field an $T$ indicates $t$-ordering. From the definition (18) of $u_{x y}^{n}$, one finds

$$
u_{x y}^{n}=1-\frac{a^{2}}{2} \sum_{\mu>v}(x+y-2 n)_{\mu}(x-y)_{\nu} F_{\mu \nu}(a n)+O\left(a^{3}\right) .
$$


It follows to leading order as $a \rightarrow 0$, that only the plaquette terms contribute to the charge density:

$$
\begin{aligned}
q(n)= & \frac{1}{8 \pi^{2}} \sum_{\mu, v, \varrho, \sigma} \varepsilon_{\mu \nu \varrho \sigma}\left\{\int_{p(n+\hat{v}, \mu, v)} d^{2} x \operatorname{Tr}\left[\partial_{\varrho} P_{n+\hat{v}, \mu \nu}^{n} \partial_{\sigma} R_{n, \mu ; \nu}^{n}\right]\right. \\
& \left.-\int_{p(n+\hat{\mu}+\hat{v}, \mu, v)} d^{2} x \operatorname{Tr}\left[\partial_{\varrho} P_{n+\hat{\mu}+\hat{v}, \mu \nu}^{n} \partial_{\sigma} R_{n+\hat{\mu}, \mu ; \nu}^{n}\right]\right\} .
\end{aligned}
$$

From this, Eq. (34) and the definitions of the $P$-and $R$-matrices, one immediately infers that

$$
q(n)=a^{4} \sum_{\mu ; v, \varrho, \sigma} \tau_{\mu \nu \varrho \sigma} \operatorname{Tr}\left\{F_{\mu \nu}(a n) F_{\varrho \sigma}(a n)\right\}+O\left(a^{5}\right)
$$

where $\tau_{\mu \nu \varrho \sigma}$ is some tensor independent of $n$ and $a$. The actual computation of $\tau_{\mu \nu \varrho \sigma}$ is tedious but presents no difficulty and is therefore omitted here. As asserted in Sect. 1, the outcome is Eq. (6). Thus, for small lattice spacings (relative to the wave lengths of the classical field), the lattice topological charge is equal to the continuum topological charge (1).

\section{Concluding Remarks}

Almost all fields contributing to the functional integral of any (continuum) quantum field theory in $d \geqq 2$ space-time dimensions are rather discontinuous (e.g. [6]). It is surprising, therefore, that topological structures, which assume continuous or even differentiable fields, are sometimes not totally washed out in the functional integral. In the case of the non-Abelian gauge theories with a lattice cutoff, the reason is that due to asymptotic freedom (i.e. $g \rightarrow 0$ in the continuum limit), the dominant fields acquire some continuity at scales of the cutoff, which is already sufficient to divide the field space into disconnected pieces.

An interesting question not addressed in this paper is whether or not the sectors $Q \neq 0$ are statistically relevant (this is the infrared aspect of the problem). It can be answered, for example, by studying the topological susceptibility $\chi_{t}$ [Eq. (2)]. In the continuum limit $g \rightarrow 0, \chi_{t}$ should obey the renormalization group formula

$$
\chi_{t}=k \Lambda^{4}\left\{1+O\left(g^{2}\right)\right\}
$$

where $k$ is a numerical constant and

$$
\Lambda=a^{-1}\left(g^{2}\right)^{-\frac{51}{121}} \exp -\frac{12 \pi^{2}}{11 g^{2}}
$$

is the standard of mass at $g=0$. If $k \neq 0$, topological effects (e.g. $\theta$-vacua) will show up, and if $k=0$, they will be absent (there is also the more complicated possibility that the scaling law (37) fails: see [4] for a detailed discussion). For a straightforward Monte Carlo evaluation of $k$, the expression (32) for the topological charge 
density is probably too complicated, unless a parallel computing facility is available. As one is only interested in the total charge, however, there may be more efficient combinatorial methods to compute $Q$ for any given lattice gauge field.

\section{References}

1. Belavin, A., Polyakov, A., Schwartz, A., Tyupkin, Y.: Phys. Lett. 59B, 85 (1975)

2. Di Vecchia, P., Nicodemi, F., Pettorino, R., Veneziano, G.: Nucl. Phys. B181, 318 (1981)

3. Wilson, K.G.: Phys. Rev. D14, 2455 (1974); for reviews see e.g.

Kadanoff, L.P.: Rev. Mod. Phys. 49, 267 (1977)

Drouffe, J.M., Itzykson, C.: Phys. Rep. 38C, 133 (1978)

4. Berg, B., Lüscher, M. : Nucl. Phys. B190, [FS], 412 (1981)

Lüscher, M. : Nucl. Phys. B200, [FS4] 61 (1982)

5. Kobayashi, S., Nomizu, K.: Foundations of differential geometry, Vol. I. New York: Interscience 1963

6. Colella, P., Lanford, O.E., III: Constructive quantum field theory. Velo, G., Wightman, A. (eds.). In: Lecture Notes in Physics, Vol. 25. Berlin, Heidelberg, New York: Springer 1973

Communicated by R. Jost

Received June 22, 1981 